\title{
Bistability in Ephaptic Coupling as a Possible Memory Element Explored through a Colloid Interptation of Stereocilia Bistability
}

\author{
Michelangelo Rossetto \\ 125 w 86 th st, New York, USA \\ Email: marossetto@libero.it
}

Received 14 January 2015; accepted 22 March 2015; published 25 March 2015

Copyright (C) 2015 by author and Scientific Research Publishing Inc.

This work is licensed under the Creative Commons Attribution International License (CC BY). http://creativecommons.org/licenses/by/4.0/

(c) (i) Open Access

\begin{abstract}
When the processes of neurons are in close proximity they effectively couple to each other in a process termed Ephaptic coupling. This coupling occurs without the membranes touching when the space between them is some tens of nanometers. If the intra-membrane spacing has a mechanical bistability, which moves the membranes closer and further apart, the Ephaptic coupling will be turned on and off allowing one neuron to read, or not read the content of the other. This paper explores the possibilities of bistable ephaptic memory element by a study of an analogous system that operates at a much larger scale, the Stereocilia of the hair cell. Published measurement of hair cell Stereocilia force/displacement function shows both a negative slope region in the displacement function and tendency to express bistability. We show here how this negative slope region can arise through the exigency of colloid forces. An explanation of Colloid Theory, presented in a graphic form, shows how a colloid force function can be modified to match the measured hair cell cilia force function. The colloid force function is modified by a limiting function resulting from Stereocilia side links, structural details that tie together the hair cell Stereocilia clusters. Understanding how a limited, simple behavior such as Stereocilia bistability functions may point to a more general understanding of how bistability may underlie other areas of living organisms such as memory and computation.
\end{abstract}

\section{Keywords}

Ephaptic, Hair Cell, Stereocilia, Colloid, Bistability, Memory

\section{Introduction}

Ephaptic interactions in neural networks are now recognized as a significant modality that must be incorporated

How to cite this paper: Rossetto, M. (2015) Bistability in Ephaptic Coupling as a Possible Memory Element Explored through a Colloid Interptation of Stereocilia Bistability. Journal of Electromagnetic Analysis and Applications, 7, 107-114. http://dx.doi.org/10.4236/jemaa.2015.73012 
in the understanding of complex neural systems [1] [2]. This paper explores the possibilities that arise from bistability that is integrated into an ephaptic interaction. This bistable/ephaptic interaction, where the bistability moves the membranes involved closer and further apart, has the potential to serve as a memory element.

The importance of bistability in biology has been long recognized. Bistability plays a role in cell division, differentiation, cancer onset and apoptosis. These forms of bistability occur when a cell chooses one of two possible developmental paths depending on local conditions. They are slow acting. Time is measured in hours and days.

The colloid driven bistability we consider here is fast. It operates in microseconds. Colloid forces are electromagnetic forces resulting from the physical chemistry of cellular surfaces. A colloid force function results from the interaction of attractive and repulsive forces. The attractive forces are Van der Wall interactions where the constituent molecules are polarized. Repulsive forces result from the negatively charged electron clouds of nearby particles repelling each other. Figure 5(a) illustrates how the attractive/repulsive interaction results in the colloid function. The attractive and repulsive forces are inverse power functions with different exponents. Their interaction is seen to result in a function that has areas with both positive and negative slopes.

Measurement in university labs of colloid forces in the 1980s' uncovered the shape of the colloid function. It was, however, bedeviled by bistability during the measurements [3]. This bistability arose when the measuring probe presented a light load to the colloid system. In Figure 5(b) the jumps J and J' are expressions of the bistability that results from the negative slope region of the force curve. The part of the force curve that has a negative slope corresponds to the hump in the energy curve that separates two areas of minimum energy which are stable resting places. An exhaustive review of colloid measurement techniques and their difficulties was written by Craig in 1997 [4]. Israelachvilli wrote the definitive text on the subject of colloid interactions [5] [6].

Emulsions are colloid driven suspensions of, for example, fat droplets suspended in water or water droplets suspended in fat. They are stable when the interaction of the two substances rests in an energy minimum. Cosmetics and many food products are colloidal emulsions. Butter, for example, is a water in fat emulsion, while milk is a fat in water emulsion.

One of the earliest recognition of a colloidal system in a living biological function was the observation that clusters of vesicles found at nerve terminal synapse behave like an emulsion. A typical study of this phenomenon is found in a 1981 paper by Nir. S. from which the energy curve of Figure 5(a) was abstracted.

The vesicles clustered in nerve terminals adjust their spacing as an expression of their inter vesicle colloid interaction. Although the vesicles are spherical, when one considers the cross section formed by the common equatorial plane of two adjacent vesicles it is obvious that the cross section is the same as the cross section of two adjacent hair cell Stereocilia as shown in Figure 3. They both have similar dimensions, are bounded by similar membranes and have similar spacing [7]. These similarities are what lead to the consideration of hair cell Stereocilia to be working under the same influence of colloid forces as are the vesicles.

Two measurements, selected from Hair Cell literature, are used here in support of a theory of a colloid based bistable functionality in a living biological system. These two measurements are both quantitative. They are:

1) The measurement of a negative slope in the force/distance function of cilia which are accompanied by a mechanical bistability.

2) The measurement of the separation limit of adjacent cilia due to cilia side links which behave like a "door chain" that allows only limited separation.

We show how these measurements, taken together and integrated into a graphically presented colloid interaction, illustrate a plausible mechanism for the mechanical bistability observed in the Hair Cell Cilia and hypothesized for an ephaptic bistability.

\subsection{Force/Distance Measurement}

Martin measured the mechanical force versus distance function of hair cell cilia [8]. As shown in Figure 1, this measured curve shows that the hair cell cilia have an area of negative slope in their force versus distance function

These force measurements were made difficult by mechanical jumps, or bistability during the measurement. The area of negative slope can underlie the bistability observed in the hair cell cilia. Martin pointed out that this curve mimicked the characteristic curve of the tunnel diode, shown in Figure 4, and he suggested that knowledge accumulated in the study and application of the tunnel diode could be useful in interpreting hair cell activity. The tunnel diode can be used in electronic bistable circuits, electronic circuits that have two stable states. 


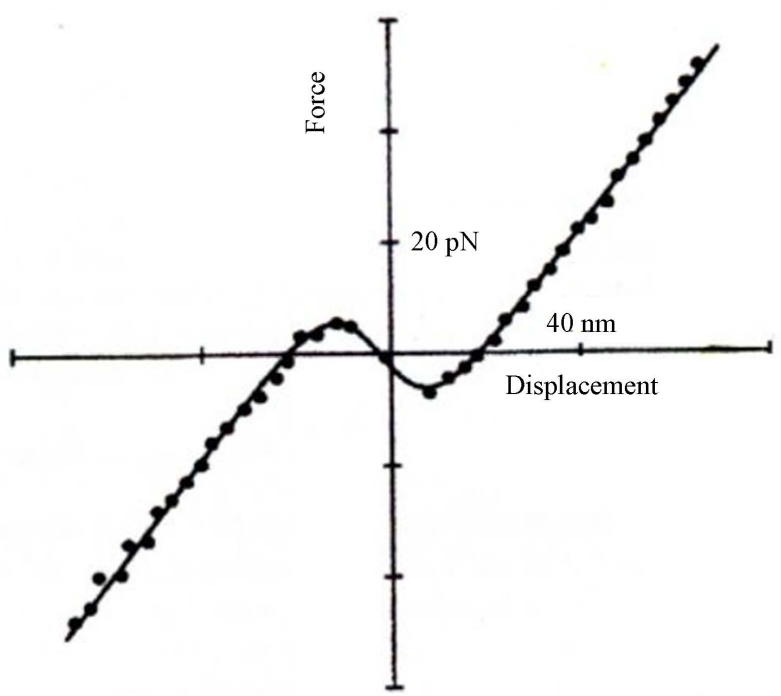

Figure 1. A simplified curve abstracted from the "force-displacement" function of the "cilia-kinocilium" bundle from in vitro hair cell measurements performed and presented by Martin [8]. This measurement uncovered a negative slope portion of the curve. It locates the active behavior of the hair cell, which includes bistability at the ciliated end of the cell. This observation led Martin to point out the functional similarity of the hair cell cilia and the operating curve of the Tunnel diode, an electronic semiconductor device. The negative slope part of the curve spans a distance of approximately $30 \mathrm{nM}$ and a force range of about $20 \mathrm{pN}$.

\subsection{Side Links Limit Cilia Separation}

Nam studied the extra-cellular links in the vestibular hair bundle. Figure 2 and Figure 3 show the cilia side links schematically.

These links constrain, or limit, to nanoscale dimension the spacing between cilia. After careful measurements Nam stated that the side links experience zero tension when they are slack. When they reach the limit of their extendibility they rigidly prevent any further increase in the spacing between cilia, just as a door chain does [9]. Nam summarized this behavior graphically and I have redrawn it, with a scale based on his measurements and the dimensions in Martins measurements, in Figure 5(c).

\subsection{Bistability in the Tunnel Diode}

When the tunnel diode [10] is biased with the constant current (I bias) it has the two stable voltage states, $\mathrm{X}$ and Y, which express its bistability as shown in Figure 4. Martins measured "force-displacement" curve of Hair Cell Cillia, [8] which contained a segment with a negative slope, can be expected to function in its mechanical domain with a bistability in a manner analogous to the electrical behavior of the tunnel diode.

\section{Model Theory}

By showing how a colloid force function can be modified by the limiting function resulting from cilia side links we demonstrate how a new function is formed. This function has the same form as Martins measured cilia function and has the same form as the tunnel diode curve.

The theory of hair cell activity presented here is based on the area of physical chemistry that deals with colloid interactions. Interactions between colloid particles are an electromagnetic phenomenon. The combined van der Waal attractive dipole forces and the electrostatic repulsion experienced by the orbiting electrons of adjacent particles as they approached each other underlies colloid interactions. This model considers the cilia of hair cells to be colloid particles whose electromagnetic surface interactions underlie the bistable behavior observed in the course of Hair Cell Cilia measurements. It describes the requirements for a device that marshals colloid forces in 


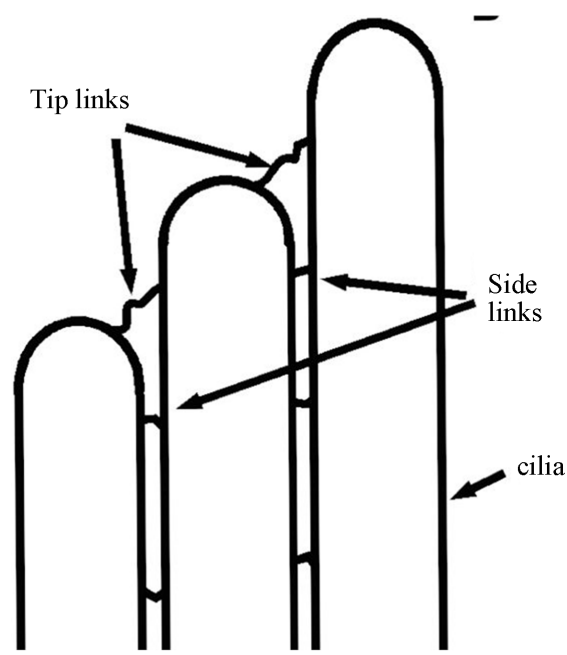

Figure 2. A schematic side view of adjacent cilia showing the typical placement of the side links that keep the cilia from moving too far apart.

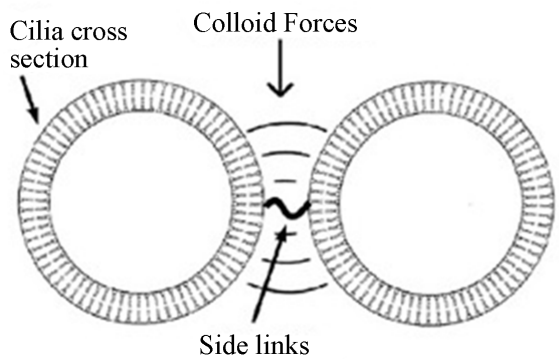

Figure 3. A schematic cross sectional view of two adjacent cilia showing how the side links limit the spacing between cilia and how the colloid forces activate the inter cilia interactions. This figure expands on Figure 2 of [7] by the inclusion of the side links.

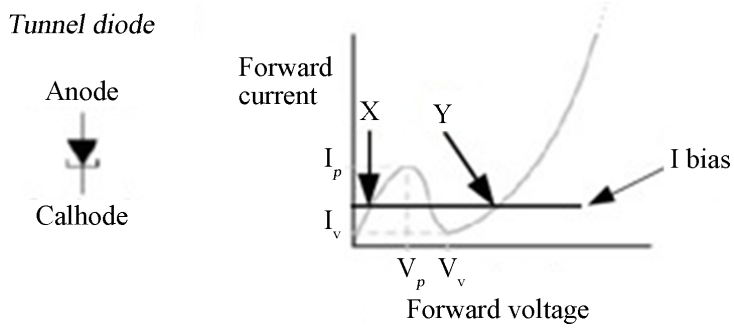

Figure 4. The functional curve of the tunnel diode, an electronic device which can be used as an amplifier, oscillator, or bistable memory element. Martin pointed out its similarity to the measured hair cell force curve. When the tunnel diode is biased with a constant current, "I bias" in this figure, the diode has two stable states. These two stable states are labeled $\mathrm{X}$ and $\mathrm{Y}$ in the figure.

the realization of an active mechanical device which exhibits a mechanical bistability that can exist in a living biological organism. Background and a historical perspective of the application of colloid theory to hair cell cilia can be found in a previous paper [7].

\subsection{Colloid Energy}

Figure 5(a) shows both Vw, the van der Waal attractive force, and Ve, the electrostatic repulsive force. They are 
both inverse power functions with different exponents in the energy domain. The van der Waal forces are the attraction resulting from dipole moments. The electrostatic repulsive forces result from the orbital electrons of adjacent particles getting close enough to feel each other. The interaction of the attractive van der Waal and repulsive electrostatic forces is equivalent to a subtraction of two similar large numbers. This is a process that emphasizes small differences. In this case the subtraction of the differing ripples produced by the two inverse power functions with different exponents, results in the complex shape of the resulting combined energy function Vt of Figure 5(a).

A change of the distance between two colloid particles will subject them to energy changes of the type shown in Figure 5(a), which is adapted from a study of vesicles [6]. This figure presents in simple graphic form the DLVO energy relations that determine the basic behavior of a typical lyophobic colloid system. The abscissa (x) is separation, with zero distance, or contact, at the left of the figure. Vt is the resultant energy of interaction which combines Van der Waals attraction Vw and the electrostatic repulsion Ve.

\subsection{Colloid Forces}

Figure 5(b) presents the slope of the energy curve Vt, dV/dx, which is the force experienced by closely opposed colloid particles. Colloid particles will congregate in an energy minima if it is deep enough, that is deeper than the thermal energy of the particles [6]. Note that at the energy minimum the force is zero. This is a stable resting place on the force curve.

The part of the Force curve important to a colloid theory of the hair cell, is the part of the curve of Figure 5(b) that has a negative slope. The part of the force curve that has a negative slope is like the negative portion of the measured cilia force function shown in Figure 1. Force curves similar to the curve of Figure 5(b) have often been drawn from careful measurements of colloid systems. Craig presents a review of the field of colloid force measurements, the difficulties and the instruments used to make them [4].

\subsection{Limiting Function}

The links between cilia limit the separation that is possible between these elements. This limiting of the inter cilia spacing described by Nam [9], is represented by the curve of Figure 5(c). When the limit function is combined with the force function, the limited force function will be as shown in Figure 5(d). The placement of the limiting function breakpoint is consistent with the quantitative measurements listed in the results section.

\subsection{The Limited Force Function}

Figure 5(d) shows how the limit function modifies the colloid force function.

When the cilia are close to each other the links between adjacent colloid particles are slack. The forces they experience are the Colloid forces. When the links are stretched to their limit, the Limit Function quickly dominates. The result is that the Limited Force Function, shown in Figure 5(d), now resembles both the form of the tunnel diode curve of Figure 4 and the form of the measured force-distance curve of the cilia of the hair cell of Figure 1. The Points labeled A and B in Figure 5(d) are the two stable states that underlie the bistable functionality. They represent two stable points in the Limited Force curve.

\section{Results}

The limiting of the cilia spacing, or more generally, the limiting of the spacing between colloid elements, is the key to the concept of colloid mediation of the bistability as seen in hair cell cilia.

After careful measurements Nam stated that the side links experience zero tension when they are slack. When they reach the limit of their extendibility they rigidly prevent any further increase in the spacing between cilia, just as a door chain does. Nam [9] found the length of the links when fully extended, and therefor the break point for this limiting action is $78 \mathrm{nM}$ in the system he studied. The break point of $78 \mathrm{nM}$ fits quite well with the measured active range of $30 \mathrm{Nm}$ measured by Martin in the Hair Cell.

\section{Discussion}

Laboratory measurement of colloid force functions reported by Craig [4] and Horn [3] often showed bistable 


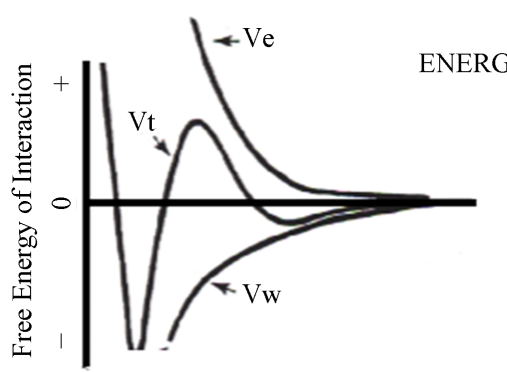

(a)

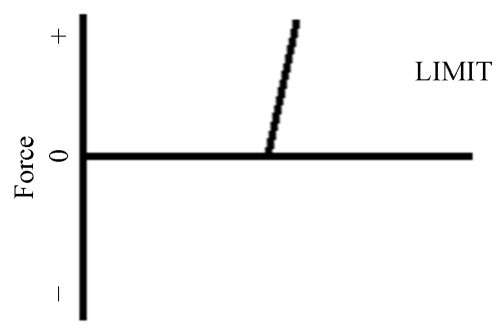

(c)

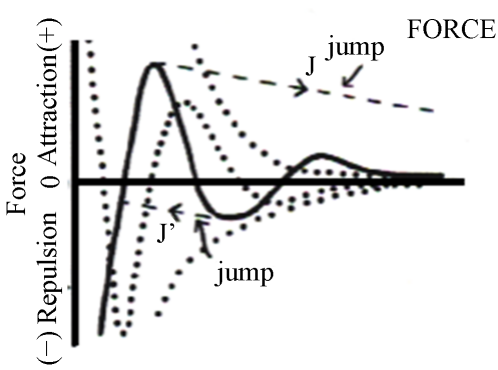

(b)

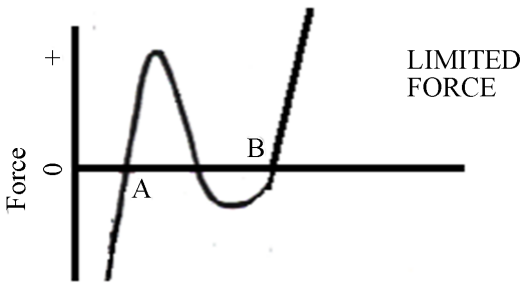

(d)

Figure 5. (a) this figure (first presented as Figure 3A in ref [6] is a summery of basic colloid theory showing the free energy of interaction Vt that results from the combined Van der Walls attraction, $\mathrm{Vw}$, and the electrostatic repulsion, Ve, experienced by adjacent colloid particles. The Van der Wall force, $\mathrm{Vw}$, is the consequence of the attraction of molecular dipoles in adjacent particles. The electrostatic repulsion, Ve, arises when the orbital electrons of adjacent particles get close enough to feel each other. The abscissa, $\mathrm{X}$, represents the distance between the colloid particles. Contact, zero distance, is at the left of the figure. (b) The slope of the energy curve Vt, $\mathrm{dV} / \mathrm{dx}$, which is the force experienced by adjacent colloid particles as a function of separation (x) was drawn from Figure 3B of ref [7]. It represents a graphic summery of the colloid force relations that are explored in this paper. Note that at the energy minimum of (a), the force curve, shows a stable resting place where the colloid particles experience neither repulsion nor attraction. (c) The side link behavior expressed graphically. Showing how the links are like the ropes that tie a boat to a dock. When the rope is slack there is no force in the rope, when the boat moves away from the dock, the rope reaches its limit and becomes tense. The swiftly rising curve limits how far apart the cilia are to each other. (d) The combined interaction of the colloid force function and the limit function showing how the resulting combined function has the same form as the measured cilia force function of Figure 1 and the tunnel diode curve of Figure 4.

behavior when the colloid system was lightly loaded by the measuring apparatus. This bistability is shown by the jumps $J$ and $J$ ' in the Force function of Figure 5(b).

Martin's measurements of the hair cell cilia force function shown in Figure 1 were irritatingly accompanied by bistable jumps during the measurements. Bistable behavior is seen in lightly loaded Hair Cell cilia [8]. Notice that in Martin's curve there are no data points in the section with a negative slope. This is the unstable zone which engenders jumps from one stable zone to the other.

Cillia functional similarity to the tunnel diode operating curve includes bistability. Martin reported his observations of a "tunnel diode like" curve and bistability but did not have an attempt to define how the organism achieves this result. This is the question this paper addresses. Is the mechanism behind bistability in the hair cell natures use of colloid forces? By bringing together hair cell measurements reported in the biophysical literature and colloid theory this paper presents arguments to support a colloid view of hair cell cilia functionality.

While the theory of colloid mediated bistability is developed here to explain the observed behavior of the Hair Ccll Cilia, the process described is not limited to the Hair Cell Cilia. Colloid forces will develop whenever two cellular membranes have the appropriate close spacing. If the membranes of adjacent neurons or their processes are close enough to experience colloid forces and are tied together with a limiting link, similar to the side links seen between Hair Cell Cilia, a spatial bistability will appear between these membranes. This spatial bistability would be well placed to modulate Ephaptic coupling between neurons [11]. 
The bistability observed in Stereocilia operates in a dimensional space where microns are the measure of Stereocilia dimensions. The area available for a colloid interaction when two dendrites pass closely is limited. This area will be orders of magnitude smaller than the Hair Ccll Stereocilia area involved in a colloid interaction. Yet this small patch is just as capable of bistability as is the Hair Cell if the spacing is appropriate and there is a limiting fiber. There are hints of fibers spanning the space between adjacent dendritic membranes in some early SEM images [12] [13].

If this small area common to two passing dendrites is functioning as a Ephaptic coupling and is at the same time bistable we have a unitary memory element. The two bistable states allow either an ephaptic coupling or no coupling.

If nature has chosen to fill it's heed for a resettable memory element by exploiting the bistability inherent in a colloid system, a two state ephaptic coupling mechanism may be a contender.

\section{Conclusions}

If the Stereocilia of the hair cell are indeed behaving as a colloid system, as the data quoted here support, it is a model for a process that may be present in areas of an organism that need memory and computation.

This paper presents a theory of Hair Cell functionality that is based on published biophysical measurements and Colloid Theory. It builds on a summary of both hair cell structure and basic Colloid theory that can be found in an earlier paper [7], which showed how colloid theory could be applied to understanding some early hair cell measurements.

The importance of this paper is twofold.

First it demonstrates that published experimental measurements, considered individually, may not lead to an explanation of the process being measured. A perspective that allows for the simultaneous view of the results of disparate work, either in the same field or in different fields, may engender novel insights. By considering Biophysical measurements in conjunction with colloid theory new insights unfold.

Secondly by recognizing that a phenomenon that is expressed on two grossly disparate scales can be studied in the more accessible larger scale. Bistability that is measurable in Hair Cell cilia can give insight toward the understanding of the less accessible behavior of a possible Ephaptic bistability. An Ephaptic colloid bistability memory element, if it exists, would allow not only remembering but also forgetting. A colloid driven bistability could be both set and reset by the attraction and repulsion of nearby electric fields. Colloid forces are electromagnetic forces.

\section{A Testable Prediction}

If the vibratory motion of the cilia bundle is operating under the influence of the electro magnetic forces of a colloid system it should be susceptible to external magnetic fields. This makes it a candidate for the magnetic sensitivity which underlies the compass used by migrating animals. A test for this sensitivity could be made by observing the resting discharge of, for instance, a lateral line hair cell stitch as the surrounding magnetic field is rotated.

\section{References}

[1] Vroman, R., Klassen, L. and Kamermans, M. (2013) Ephaptic Communication in the Vertebrate Retina. Frontiers in Human Neuroscience, 7, 612.

[2] Anastassiou, C.A. and Koch, C. (2014) Ephaptic Coupling Endogenous Electric Field Activity: Why Bother? Current Opinion in Neurobiology, 31, 95-103.

[3] Horn, R.G. and Israelachvilli, J.N. (1981) Direct Measurement of Structural Forces between Two Surfaces in a Nonpolar Liquid. The Journal of Chemical Physics, 75, 1400-1411. http://dx.doi.org/10.1063/1.442146

[4] Craig, V.S. (1997) A Historical Review of Surface Force Measurements Techniques. Colloids and Surfaces A, 129-130, 75-93. http://dx.doi.org/10.1016/S0927-7757(97)00029-0

[5] Israelachvilli, J. (1985) Intermolecular and Surface Forces. Academic Press, London.

[6] Nir, S.J., Bentz, J. and Duzgunes, N. (1981) Two Modes of Reversible Vesicle Aggregation: Particle Size and the DLVO Theory. Journal of Colloid and Interface Science, 84, 266-269.

http://dx.doi.org/10.1016/0021-9797(81)90284-8 
[7] Rossetto, M. (2003) Colloid View of Hair Cell Cilia: A Selective Review. Colloid and Surfaces B, 29, 257-263. http://dx.doi.org/10.1016/S0927-7765(03)00004-3

[8] Martin, P., Mehta, A.D. and Hudspeth, A.J. (2000) Negative Hair-Bundle Stiffness Betrays a Mechanism for Mechanical Amplification by the Hair Cell. Proceedings of the National Academy of Sciences of the United States of America, 97, 12026-12031. http://dx.doi.org/10.1073/pnas.210389497

[9] Nam, J., Cotton, J., Peterson, E. and Grant, W. (2006) Mechanical Properties and Consequences of Stereocilia and Extracellular Links in Vestibular Hair Bundles. Biophysical Journal, 90, 2786-2785. http://dx.doi.org/10.1529/biophysj.105.066027

[10] Roy, D.K. (1997) Tunneling and Negative Resistance Phenomena in Semiconductors. Pergamon Press, Elmsford.

[11] Anastassiou, C.A., Perin, R., Markram, H. and Kich, C. (2011) Ephaptic Coupling of Cortical Neurons. Nature Neuroscience, 14, 217-223. http://dx.doi.org/10.1038/nn.2727

[12] Gulley, R.L. and Rees, T.S. (1981) Cytoskeletal Organization at the Postsynaptic Complex. The Journal of Cell Biology, 91, 298-302.

[13] Landis, D.M. and Reese, T.S. (1983) Cytoplasmic Organization in Cerebellar Dendritic Spines. The Journal of Cell Biology, 97, 1169-1178. 